\title{
Aplicación de la técnica fotoacústica resuelta en tiempo al monitoreo de la fotosíntesis en plantas de lirio acuático (Eichhornia Crassipes)
}

\author{
A. Cardona $^{1} \mid$ R. Abdelarrague ${ }^{1}$ G. Peña Rodríguez ${ }^{2}$ E. Marín ${ }^{1} \mid$ A. Calderón ${ }^{1 *}$
}

Recibido:

27 de marzo de 2008

Aceptado:

1 de junio de 2008

\section{Resumen}

Reportamos el uso de la técnica fotoacústica resuelta en tiempo en el análisis de la evolución de oxígeno fotosintético y el almacenamiento de energía en plantas verdes. Presentamos una aplicación de esta técnica al monitoreo de la fotosíntesis en la maleza acuática llamada comúnmente lirio acuático o jacinto de agua (Eichhornia Crassipes).

Palabras Claves: Fotosíntesis, técnica fotoacústica, lirio acuático.

\section{Abstract}

We reported about the use of the time resolved photoacoustic technique for the analysis of the photosynthetic oxygen evolution and the energy storage in green plants. We present an application of this technique to the study of the photosynthesis in the aquatic vegetation, usually called aquatic lirium or water hyacinth (Eichhornia Crassipes).

Keywords: Photosynthesis, photoacoustic technique, water hyacinth.

\section{Introducción}

${ }^{1}$ Centro de Investigación en Ciencia Aplicada y Tecnología Avanzada Unidad Legaria del Instituto Politécnico Nacional, Legaria 694 Colonia Irrigación, 11500 México D. F.

${ }^{2}$ Departamento de Física, Universidad Francisco de Paula Santander. A.A. 1055, Cúcuta, Colombia.

*e-mail: jcalderona@ipn.mx 
posterior análisis de la información obtenida. Dependiendo del tipo de detector empleado para realizar la transformación de la respuesta térmica del material en una señal eléctrica, es el nombre que se le asigna a la técnica de medición. De esta manera, cuando se utiliza un detector acústico, la técnica se denomina fotoacústica, si el detector es un piroeléctrico, la técnica se denomina fotopiroeléctrica, cuando se usa un detector óptico, la técnica se designa radiometría fototérmica [1].

En la técnica fotoacústica (FA) se pueden tener, en general, tres tipos de esquemas de medición que dependen del parámetro físico que se hace variar para determinar la señal FA como una función de este parámetro. El primero, es aquel en el cual se hace variar la frecuencia de modulación de la fuente luminosa, que mediante el análisis de la amplitudy la fase dela señalFA medida, permite determinar parámetros de transporte de calor, así como tiempos de relajación y velocidades de recombinación de portadores, entre otras propiedades de la muestra en estudio [2-4]. El segundo caso, es aquel que manteniendo constante la frecuencia de modulación de la fuente, se hace variar la longitud de onda de la fuente de luz incidente sobre la muestra, entonces tratamos con la técnica FA resuelta en longitud de onda, también denominada espectroscopia fotoacústica (EFA), la cual permite obtener espectros de absorción ópticos de la muestra [5-7]. La tercera configuración, es aquella en la cual se mantienen constantes tanto la frecuencia de modulación como las características de la fuente de radiación (longitud de onda e intensidad) y el parámetro que varía es el tiempo de exposición, la cual se conoce como la técnica FA resuelta en tiempo. Esta técnica permite estudiar, entre otras cosas, la evolución de oxígeno fotosintético y el almacenamiento de energía en plantas [8].
Lirio acuático, jacinto de agua, camalote, lampazo, violeta de agua, buchón o taruya, entre otros, es el nombre vulgar que se le da a la planta acuática con nombre científico Eichhornia Crassipes. Es una planta libre flotadora, perteneciente a la familia de las Pontederiáceas. Originaria de América del Sur (Amazonas), la que por la belleza de su flor se ha propagado a casi todas las áreas tropicales y sub-tropicales del mundo. Uno de los factores que pueden limitar su propagación es la salinidad, ya que no tolera el agua salobre, por lo que serán los cuerpos de agua continentales y mixohalinos donde se encuentra dicha especie. Su rápida reproducción, así como la ausencia de enemigos naturales en los nuevos lugares de su introducción, además de su excelente capacidad de adaptación a casi cualquier cuerpo de agua, han provocado la rápida diseminación de la planta, convirtiéndose así en una maleza. Esto ha traído como consecuencia que diversas actividades económicas importantes se vean afectadas sensiblemente en las áreas invadidas por esta planta. Una extensa cobertura del lirio acuático provoca una evapotranspiración tres o cuatro veces superior a la que normalmente ocurre en superficies de agua libre, consumiendo el cuerpo de agua y ocasionando putrefacción del mismo por la obstrucción que provoca al paso de los rayos solares hacia su interior. No menos dañino es el efecto que ocasiona cuando invade los generadores de las estaciones hidroeléctricas, provocando cortes eléctricos de determinada duración hasta que los generadores son limpiados de tejidos de la planta [9]. Pero no todo es negativo, algunas de las bondades de esta planta, son el ayudar en la descontaminación de agua dulce (lagunas de oxidación), también es utilizada como fuente de biomasa en la alimentación de animales ya que posee los niveles adecuados de calcio, fósforo y nitrógeno [10-12]. 


\section{Procedimiento}

\section{experimental}

\section{Muestras}

Las plantas de lirio acuático se mantuvieron estables en el laboratorio, en el interior de un acuario-invernadero diseñado para simular y controlar las condiciones en las que sobrevive el lirio en su hábitat de procedencia (canales de Cuemanco, México D. F.). Durante este tiempo, se controlaron los parámetros fisicoquímicos del agua, la concentración de nutrientes (proporcionados mediante un fertilizante líquido), así como, la intensidad y tiempo de iluminación. En la tabla 1, se resumen las condiciones más convenientes que permitieron la sobrevivencia de las plantas in-vitro.

Tabla 1. Parámetros físico-químicos y concentración de nutrientes del agua del acuario-invernadero.

\begin{tabular}{|l|l|}
\hline \multicolumn{2}{|c|}{ Parámetros físico-químicos } \\
\hline $\mathrm{pH}$ & 6.05 \\
Temperatura & $25^{\circ} \mathrm{C}$ \\
Conductividad & $477 \mu \mathrm{S} / \mathrm{cm}$ \\
Cloro & $0.5 \mathrm{mg} / \mathrm{l}$ \\
Intensidad de la luz & 5000 lux \\
Tiempo de iluminación & $13 \mathrm{hrs}$. / día \\
\hline
\end{tabular}

\section{Concentración de Nutrientes}

Fertilizante líquido universal $15 \mathrm{~N}-30 \mathrm{P}-15 \mathrm{k} *$ $61.34 \mathrm{gr} / 1801^{\circ}$

* basado en una hidropónica

- 1801 = volumen de llenado

Utilizando estos parámetros en el proceso de adaptación, las plantas mostraron un desarrollo fisiológico favorable. En un inicio se introdujeron al acuario-invernadero 43 individuos juveniles de lirio con dimensiones promedio: Hoja $=5 \mathrm{~cm}$, Pecíolo $=10 \mathrm{~cm}$, Raíz $=11 \mathrm{~cm}$, procedentes de los canales de Cuemanco, México D.F. Durante 2 meses se observó el comportamiento de las plantas, tiempo en el cual no se presentó necrosis de ningún tipo, y además, se presentaron nuevos brotes de estolones y el desarrollo de más pecíolos.

Las medidas experimentales se realizaron al mediodía sobre una planta completa de lirio retirada del acuario-invernadero y mantenida en un recipiente con la misma agua durante las mediciones. El sistema de medición estuvo ubicado a $50 \mathrm{~cm}$ del acuario invernadero bajo condiciones semejantes de temperatura e iluminación. Cada corrida experimental tuvo una duración de 3 minutos y medio. Después de cada medición se colocó la planta en estudio de nuevo en el interior del acuarioinvernadero. En ningún caso se aprecio daño en las plantas estudiadas durante y después de las mediciones.

\section{Celda fotoacústica}

Figura 1. Esquema de la celda FA. (a) Cuerpo de la celda FA, (b) ventana de cuarzo, (c) cámara FA, (d) detector acústico, (e) muestra (hoja de la planta), (f) portaobjetos, (g) luz modulada, (h) respuestas fototérmica y fotobárica.

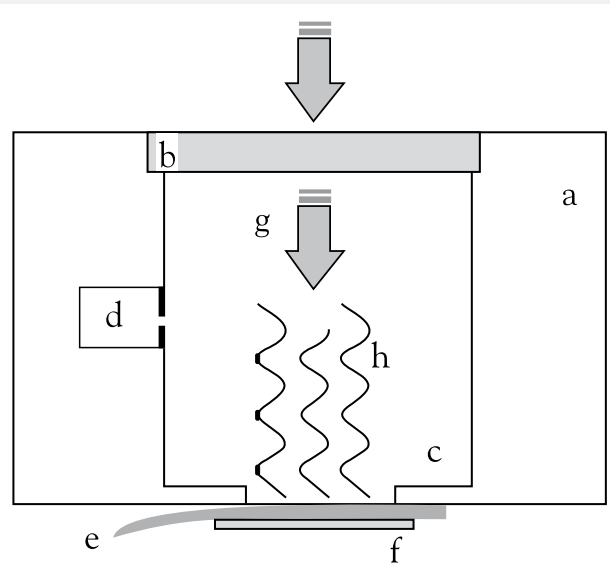

En la figura 1, se presenta el esquema de la celda FA, en la configuración de difusión de calor utilizada en este trabajo. La muestra (una hoja de la planta) se coloca adherida con grasa de vacío, en la entrada de la cámara, de manera 
que una de las caras de la muestra queda en contacto con el aire en el interior de la cámara FA y la otra descansa en un portaobjetos que la mantiene rígida sobre la base.

Si se hace pasar radiación modulada a través de la ventana de cuarzo, de manera que incida en la cara trasera de la muestra (ver figura 1), entonces, la energía luminosa absorbida por ésta genera una señal FA a la salida del transductor acústico acoplado a la celda, la cual esta constituida por la superposición de dos contribuciones:

1.-La respuesta fototérmica, que resulta de la conversión total o parcial de la luz (modulada) absorbida a calor modulado (ondas térmicas) la cual es luego transformada a una señal acústica en el aire del interior de la cámara FA.

2.- La respuesta fotobárica, debida a la evolución modulada de oxígeno producido en el proceso fotosintético, generado por la actividad fotoquímica en la hoja.

\section{Esquema de medición}

Figura 2. Diseño experimental de medición de la técnica FA resuelta en tiempo para monitoreo de la tasa fotosintética. (1) Lámpara de xenón, (2) chopper, (3) monocromador, (4) cámara FA con la muestra, (5) interfase, (6) filtro IR de agua,

(7) amplificador lock-in, (8) computador,

(9) gráfico, (10) fuente de luz continua.
El diseño experimental de la técnica FA resuelta en tiempo para la medición de la fotosíntesis en plantas se presenta en la figura 2, en ésta, un haz de luz blanca continua, proveniente de una lámpara de xenón, pasa por un modulador mecánico (chopper), cuya señal sirve de referencia para el amplificador lock-in; posteriormente, el haz modulado emergente se hace pasar por un monocromador, el cual selecciona la longitud de onda a la que se desea irradiar; así, la luz modulada se hace incidir sobre la celda FA donde previamente se ha colocado la muestra; por otro lado, un haz de la luz blanca continua incide directamente sobre la celda FA; ambos haces de luz inciden simultáneamente sobre la muestra vía una fibra óptica bifurcada; la señal obtenida de la celda FA, es leída por el amplificador lock-in, el cual filtra las señales que no se encuentren a la frecuencia de referencia y amplifica la señal proveniente de la celda FA; finalmente, la amplitud y fase de la señal es registrada y almacenada en un computador en función del tiempo de exposición.

\section{Resultados y discusión}

\section{Medición de la evolución de $\mathrm{O}_{2}$ fotosintético.}

Se utilizó una fuente de luz blanca continua de fondo suministrada por una lámpara halógena $\left(30 \mathrm{~mW} / \mathrm{cm}^{2}\right)$ y una iluminación monocromatica a $633 \mathrm{~nm}\left(12.2 \mathrm{~mW} / \mathrm{cm}^{2}\right)$ a una frecuencia de modulación fija durante la medición. Lairradiación sobre la hoja de realizó del lado del haz y alternando la exposición y bloqueo de la luz de fondo continúa cada $50 \mathrm{~s}$. En las figuras 3 y 4 se muestran los gráficos de la amplitud y fase de la señal FA en función del tiempo de exposición. 
Figura 3. Efecto negativo fotosintético en la amplitud de la señal FA.

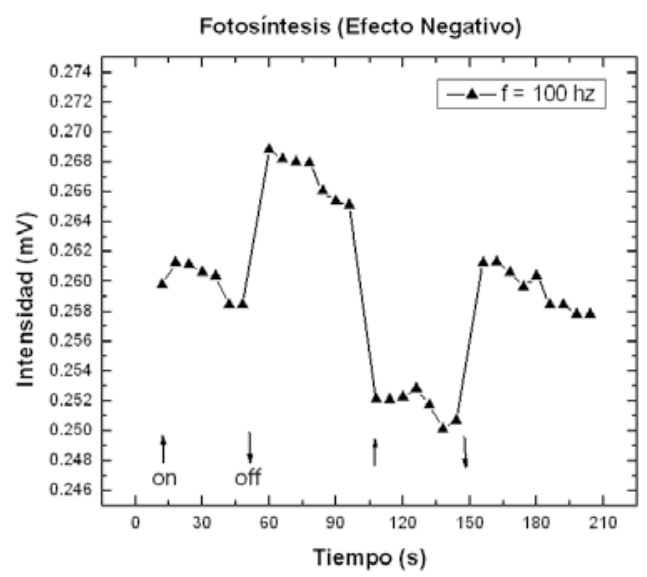

Figura 4. Efecto negativo fotosintético en la fase de la señal FA.

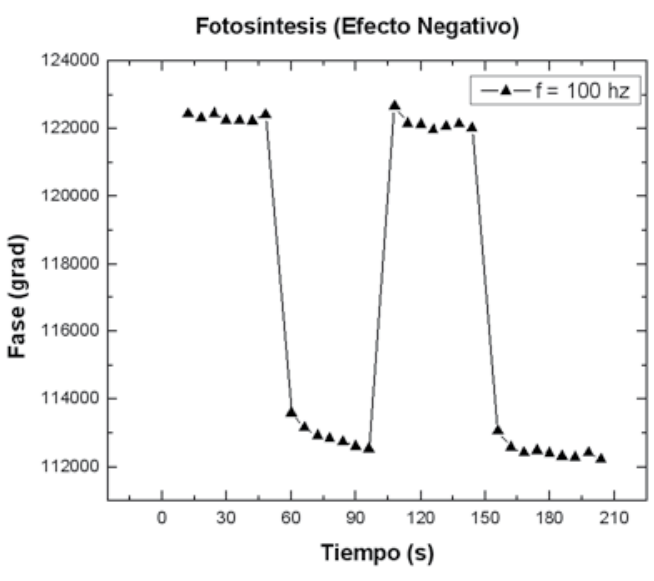

El monitoreo de la evolución de $\mathrm{O}_{2}$ se realizó mediante la medición del llamado "efecto negativo" de la señal FA. Para esto fue necesario primero determinar la frecuencia de modulación a la cual se presenta dicho efecto. Después de realizar una serie de mediciones a diferentes frecuencias de modulación, se determino que éste toma lugar a una frecuencia de $100 \mathrm{hz}$.

Considerando el valor de $1.2 \times 10^{-3} \mathrm{~cm}^{2} / \mathrm{s}$, para la difusividad térmica en la hoja [13], se determinó la longitud de difusión térmica para $f=100 \mathrm{hz}$, esto es [2]:

$$
\mu=\sqrt{\alpha / \pi f}=19.5 \mu \mathrm{m}
$$

ISSN 0122-820X

Por tanto, la onda térmica está prácticamente amortiguada a una profundidad de [8]:

$$
\lambda / 3=2 \pi \mu / 3=40.8 \mu \mathrm{m}
$$

La hoja tiene un espesor aproximado de $100 \mu \mathrm{m}$. Iniciando desde la superficie del haz hacia el interior encontramos al parénquima en empalizada, con un espesor aproximado de $30 \mu \mathrm{m}$, seguido del haz vascular con un espesor aproximado de $40 \mu \mathrm{m}$, y finalmente el parénquima esponjoso, de un espesor aproximado de $30 \mu \mathrm{m}$.

Deestamanera, lasondastérmicasgeneradas en la hoja por efecto de la incidencia del haz modulado tienen una penetración que cubre la totalidad del parénquima en empalizada y parte importante del haz vascular.

Por lo anterior, de la figura 3 se deduce lo siguiente:

Durante los primeros $50 \mathrm{~s}$, en los que la lámpara de fondo está encendida (posición "on"), no se presenta el efecto fotobárico por la saturación de los centros de reacción producida por la luz blanca continua y la señal FA es debida únicamente al efecto fototérmico. En este caso, la totalidad de la luz monocromática modulada absorbida por la hoja es transformada íntegramente en calor, una parte del cual se transmite hacia el haz vascular y el resto se difunde hacia la cámara FA. En los siguientes 50 s se suprime la luz continua de la lámpara (posición "off”), aparece la contribución fotobárica que se suma a la contribución fototérmica, y la señal FA se incrementa como se muestra en la gráfica. La evolución de oxigeno fotosintético se hace presente. En este caso, la luz monocromática modulada absorbida por la hoja es en primer 
lugar utilizada en el proceso fotosintético que da lugar como uno de sus productos a la emisión de $\mathrm{O}_{2}$, y el resto de la energía absorbida se canaliza en la generación de calor que da lugar a la contribución fototérmica. En los siguientes $50 \mathrm{~s}$ se enciende la lámpara de fondo y continúa el proceso.

\section{Estudio del almacenamiento de energía}

Se utilizaron las mismas condiciones que en el caso anterior, para la evolución de $\mathrm{O}_{2}$, excepto en la frecuencia de modulación, cuyo valor utilizado fue de $200 \mathrm{hz}$. En las figuras 5 y 6 se muestran los gráficos de la amplitud y fase de la señal FA en función del tiempo de exposición para este caso.

Figura 5. Efecto positivo fotosintético en la amplitud de la señal FA, para una frecuencia de $200 \mathrm{~Hz}$.

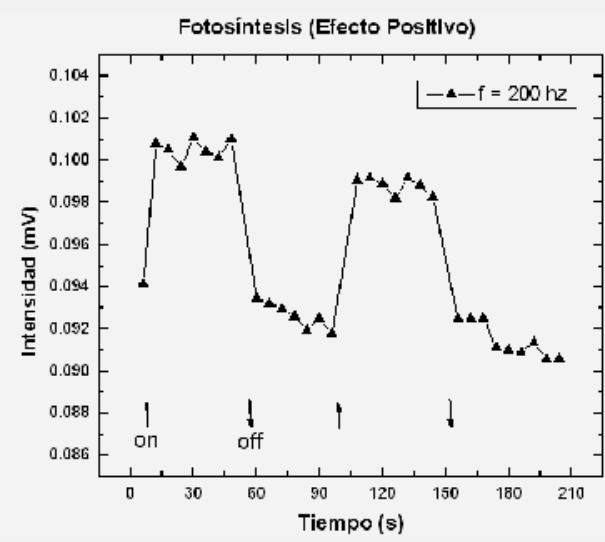

Figura 6. Efecto positivo fotosintético en la fase de la señal FA, para una frecuencia de $200 \mathrm{~Hz}$.

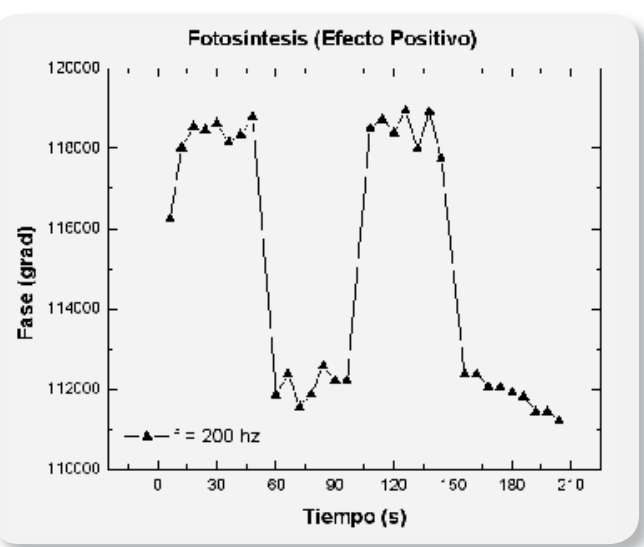

La medición del almacenamiento de energía se realiza mediante la medición del llamado "efecto positivo" de la señal FA. De nuevo, fue necesario determinar la frecuencia de modulación con la cual se presenta dicho efecto. Después de realizar una serie de mediciones a diferentes frecuencias de modulación, se determino que dicho efecto toma lugar a una frecuencia de 200 hz. Para ésta frecuencia se encuentra que la longitud de difusión térmica correspondiente es:

$$
\mu=\sqrt{\alpha / \pi f}=13.8 \mu \mathrm{m}
$$

Por tanto, la onda térmica se amortigua prácticamente a una profundidad de:

$$
\lambda / 3=2 \pi \mu / 3=28.9 \mu \mathrm{m}
$$

Deestamanera, lasondastérmicasgeneradas en la hoja por efecto de la incidencia del haz de luz modulado tienen una penetración solo en el parénquima en empalizada.

Luego, de la Figura 5 deducimos lo siguiente:

Durante los primeros $50 \mathrm{~s}$, la lámpara está encendida (posición "on"), no se presenta el efecto fotobárico por la saturación de los centros de reacción producida por la luz blanca continua y la señal FA es debida únicamente al efecto fototérmico. En este caso, la totalidad de la luz monocromática modulada absorbida por la hoja es transformada íntegramente en calor, una parte del cual se transmite hacia el parénquima en empalizada y el resto se difunde hacia la cámara FA.

En los siguientes 50 s se suprime la luz continua de la lámpara (posición "off”), la luz monocromática modulada absorbida por la hoja es en primer lugar utilizada en el proceso fotosintético y el resto de la energía absorbida 
se canaliza en la generación de calor. En este caso, la intensidad de la señal FA decrece ya que la hoja utiliza la mayor parte de la energía luminosa modulada incidente en desarrollar el proceso fotosintético, uno de cuyos productos es la generación de $\mathrm{O}_{2}$, y la parte sobrante es transformada en calor, sin embargo, la contribución fotobárica a la señal FA es pequeña comparada con la contribución fototérmica.

En los siguientes 50 s se enciende la lámpara y continúa el proceso.

\section{Conclusiones}

Se ha mostradolautilidad del procedimiento para realizar el monitoreo de la fotosíntesis en plantas utilizando la técnica FA resuelta en tiempo. Las características de esta técnica muestran la potencia y sensibilidad para realizar estudios de fotosíntesis en plantas, mediante mediciones in situ, no-invasivas, no-destructivas y no-contacto.

Laaplicación en el lirioacuático (Eichhornia Crassipes) muestra resultados de un gran detalle y claridad en su proceso fotosintético. Esto conduce a que esta metodología sea recomendada en los estudios de control en esta planta acuática.

\section{Agradecimientos}

Los autores agradecen al Programa Institucional de Formación de Investigadores del Instituto Politécnico Nacional (PIFI-IPN), así como a la Secretaría de Investigación y Posgrado del IPN por su apoyo.

\section{Bibliografía}

[1] D.P.AlmondandP.M.Patel, Photothermal Science and Techniques, Chapman \& Hall, First edition, New York, pp. 73-99. 1996.

[2] A. Calderon, R. A. Muñoz Hernández, S. A. Tomas, A. Cruz Orea and F. Sánchez Sinencio, J. Appl. Phys., 84, pp. 63276329 (1998).

[3] E. Martínez, S. García, E. Marin, O. Vasallo, G. Peña Rodríguez, A. Calderón and J. M. Siqueiros. Journal of materials Science. Vol. 39, 1233-1239 (2004)

[4] O. Vigil Galán, J. Vidal, I. Riech, G. Peña Rodríguez, A. Iribarren, J. AguilarHernández and G. Contreras Puente. Semicond.. Sci. Technol. Vol. 17, 1193 1197 (2002)

[5] A. Rosencwaig and Gersho, J. Appl. Phys, 47, 64 (1976).

[6] R. A. Muñoz Hernández, A. Calderón, A. Cruz Orea, S. Tomás, F. Sánchez Sinencio and G. Peña Rodríguez. High Temperature - High Pressures, Vol. 32, 379-383 (2000)

[7] C. Haisch and R. Niessner. Spectroscopy Europe. Vol. 14 (5). 10-15. (2002)

[8] H. Vargas and L. C. M. Miranda, Phys. Rep. 161, 43-101 (1988).

[9] John T.O. Kirk. Light \& Photosynthesis in Aquatic Ecosystems. Cambridge University Press. Second Edition. London. 1994. 
[10] I. F. Toussaint, C. Oscar Romero Cruz, L. Ruiz Ortiz y R. Gonzáles Salas. Revista Electrónica de veterinaria REDVET. Vol. VI, № 5, 1-11. (2005)

[11] Meerhoff M., Rodríguez-Gallego L. y N. Mazzeo "Potencialidades y limitaciones del uso de Eichhornia crassipes en la restauración de lagos hipereutróficos subtropicales". En: Fernández A \& G Chalar (eds.) Agua en Iberoamérica: de la limnología a la gestión en Sudamérica (pp 61-74). CYTED XVII, Buenos Aires. (2002)

[12] Valderrama, L.T. Uso de dos especies de macròfitas acuáticas (Limnobium laevigatum y Eichhornia crassipes) para el tratamiento de aguas residuales agroindustriales.UniversitasScientiarum 3. 83-98. (1996)

[13] Y. S. Touloukian, R. W. Powell C. Y. Ho and M. C. Nicolasu, Thermal Difusivity, IFI/Plenum, New York (1973). 